\title{
Breast Implants on Computed Tomography-A Pictorial Review of Normal and Pathologic Findings
}

\author{
Johannes Gossner ${ }^{1}$ \\ ${ }^{1}$ Department of Diagnostic and Interventional Radiology, \\ Evangelisches Krankenhaus Göttingen- Weende, Göttingen, \\ Germany \\ Indian J Radiol Imaging 2021;31:979-982.
}

\begin{abstract}
Address for correspondence Johannes Gossner, MD, Department of Diagnostic and Interventional Radiology, Evangelisches Krankenhaus Göttingen- Weende, An der Lutter 24, 37074 Göttingen, Germany (e-mail: johannesgossner@gmx.de).
\end{abstract}

\begin{abstract} Keywords

- breast implant

- computed tomography

- pathology

- capsular contracture

- capsular rupture

Breast augmentation with implants is frequently performed. Therefore breast implants can be seen quite frequently on computed tomography (CT) of the body. This offers the possibility to detect incidental pathology and help to improve patient outcome. Basic knowledge about imaging findings of breast implants on CT is therefore needed for radiologists reporting body $\mathrm{CT}$. CT may also be used for further workup of breast implants if magnetic resonance imaging (MRI) is contraindicated or not available. The purpose of this pictorial review is to give a brief overview of normal and pathologic findings of breast implants on CT.
\end{abstract}

\section{Introduction}

Breast augmentation with implants is frequently performed. In the United States, each year approximately 300,000 women are undergoing breast augmentation for esthetic purposes and another 88,000 women receive breast implants for breast reconstruction after cancer. ${ }^{1}$ In a population study using chest radiography, approximately $3 \%$ of Dutch women showed breast implants, ${ }^{2}$ that means, breast implants can be seen quite frequently on computed tomography (CT) of the body. This offers the possibility to detect incidental pathology and help to improve patient outcome. Therefore basic knowledge about imaging findings of breast implants on CT is needed for radiologists reporting body CT. If magnetic resonance imaging (MRI) is not possible because of contraindications (e.g., in patients with pacemakers) or access to MRI is limited in resource-poor settings, CT may be helpful in the workup of breast implants The purpose of this pictorial review is to give a brief overview of normal and pathologic findings of breast implants on chest CT.

published online

December 13, 2021
DOI https://doi.org/ 10.1055/s-0041-1735918. ISSN 0971-3026.

\section{Normal Findings}

Breast implants are either used for esthetic purposes or for breast reconstruction after breast conserving surgery with breast cancer. In cases of esthetic surgery, usually bilateral implants are used. Only in rare circumstances, like unilateral breast hypoplasia or aplasia, unilateral implants are used. In contrast, for breast reconstruction, normally a unilateral breast implant is used. Implants can be placed subglandular (i.e., between the glandular tissue and the pectoralis muscle) or submuscular (beneath the pectoralis muscle). Most surgeons prefer submuscular placement for esthetic purposes because of a more natural look and a lower rate of capsular contracture. ${ }^{1}$ On CT, the normal implant shows an oval shape and a homogeneous hypodense filling surrounded by the hyperdense implant shell (-Fig. 1). There are a lot of different implants available which cannot distinguished by CT imaging reliably. Some small infolds (undulations of the implant shell) and minimal periprosthetic fluid are considered normal findings ${ }^{3}$ (-Fig. 2 ). (c) 2021. Indian Radiological Association. All rights reserved. This is an open access article published by Thieme under the terms of the Creative Commons Attribution-NonDerivative-NonCommercial-License, permitting copying and reproduction so long as the original work is given appropriate credit. Contents may not be used for commercial purposes, or adapted, remixed, transformed or built upon. (https://creativecommons.org/ licenses/by-nc-nd/4.0/)

Thieme Medical and Scientific Publishers Pvt. Ltd., A-12, 2nd Floor, Sector 2, Noida-201301 UP, India 


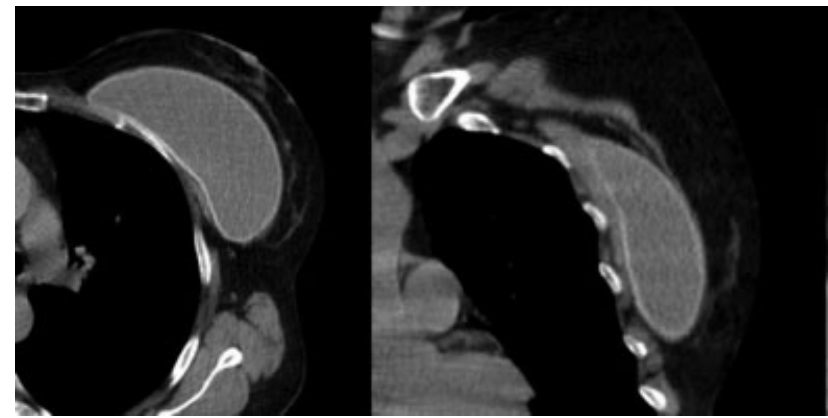

Fig. 1 Normal breast implant on computed tomography. The axial image shows the hyperdense and intact implant shell (on the left). The implant has been placed submuscular beneath the pectoralis major, which can be seen on the coronal reformation (on the right).

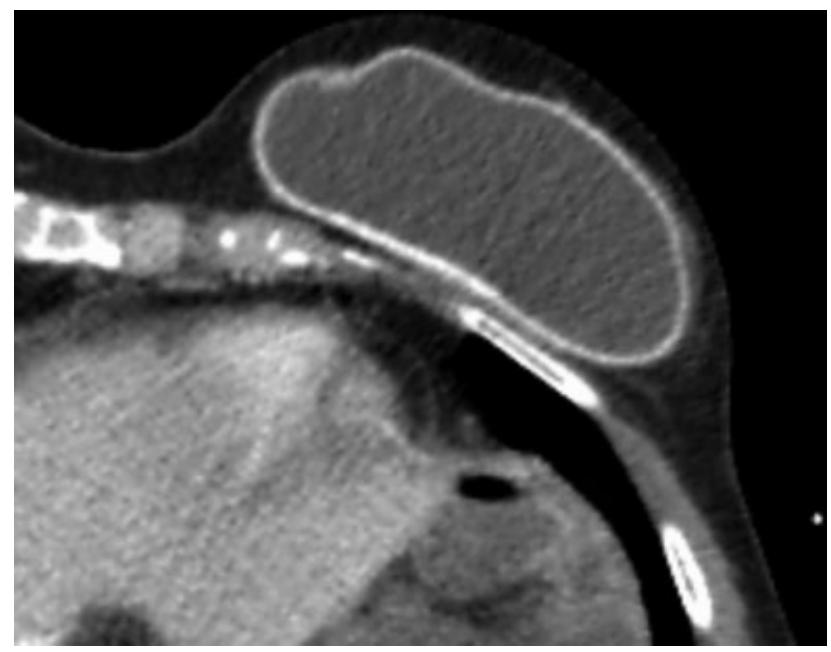

Fig. 2 Another example of a normal breast implant. The surface is showing some undulations, called infolds. This is considered a normal finding.

\section{Acute Postoperative Complications}

These include postsurgical collections (seroma and hematoma) or infection. In this scenario, usually a workup with ultrasound and image-guided aspiration is performed. ${ }^{4}$ These patients are rarely examined with CT and in these cases, there will be a dedicated clinical question concerning the implant.

\section{Capsular Rupture}

Implant rupture is common and reported to occur in up to $17.7 \%$ after primary augmentation, $14.7 \%$ after revision augmentation, and $35.4 \%$ after breast reconstruction. ${ }^{1}$ It has to be mentioned that reported rupture rates vary significantly in the literature and a large number of clinical silent ruptures are reported. ${ }^{1,3,4}$ There are two types of rupture: intra- and extracapsular. Around every breast implant, the body forms a fibrous capsule. In the case of intracapsular rupture, the filling of the implant is located inside this fibrous capsule and the collapsed shell forms the classic linguini $\operatorname{sign}^{3,4}$ (- Fig. 3). With extracapsular rupture, the implant filling leaves the fibrous capsule and migrates into the soft tissues of the breast

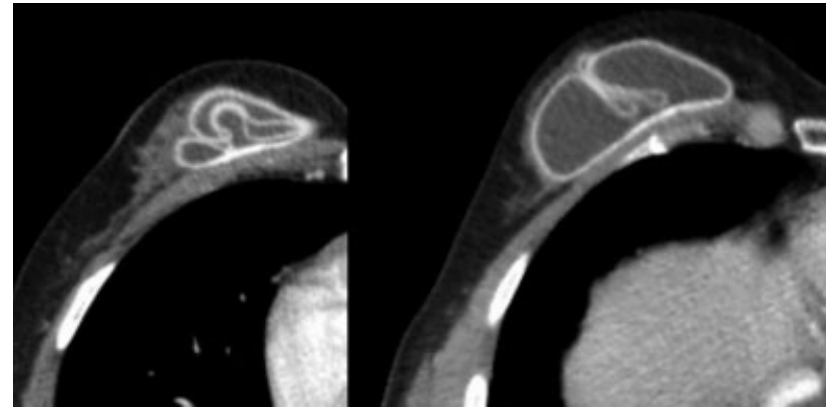

Fig. 3 Intracapsular rupture of a breast implant in a 55-year-old women undergoing chest CT because of fever of unknown origin. The collapsed shell of the implant is forming the so called "linguini-sign." $\mathrm{CT}$, computed tomography.

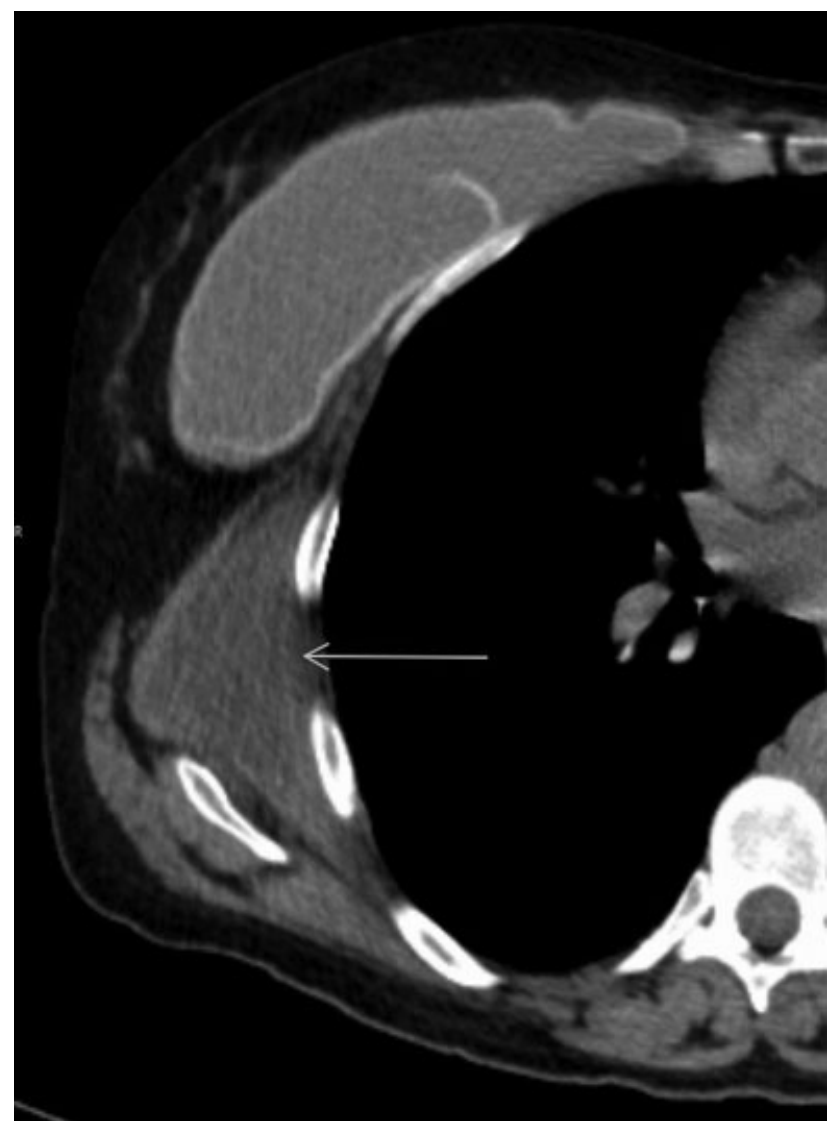

Fig. 4 Extracapsular rupture of a silicone implant in a 48-year-old women undergoing chest CT because of an enlarging mass of the thoracic wall. The implant shell is interrupted and there is leakage of the filling into the soft tissues of the thorax (arrow). CT, computed tomography.

and/or chest wall. Saline is usually absorbed by the body. In contrast, silicone forms deposits ${ }^{3,4}$ (-Fig. 4).

\section{Capsular Contracture or Fibrosis}

Capsular contracture or fibrosis is also common in the course after breast augmentation. It is reported to occur in up to $50 \%$ of women receiving breast implants, most studies are reporting incidences around $10 \%{ }^{1,5}$ In capsular contracture, the 


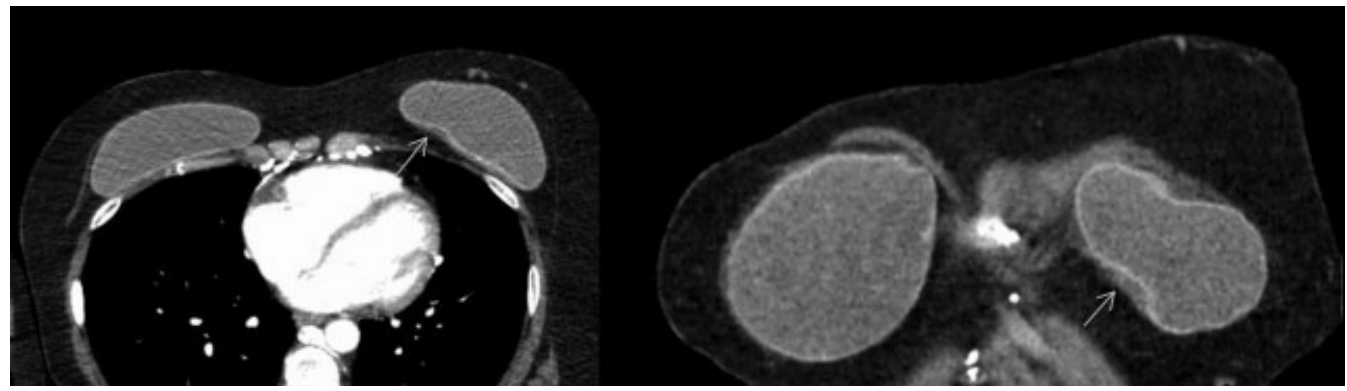

Fig. 5 Capsular contracture/fibrosis in a 44-year-old undergoing chest CT because of extensive thrombosis of the subclavian veins. The implant on the left is deformed with more infolds compared with the right-sided implant. A small band of soft tissue around the left implant can be seen, corresponding to visible parts of the thickened fibrous capsule (arrows). CT, computed tomography.

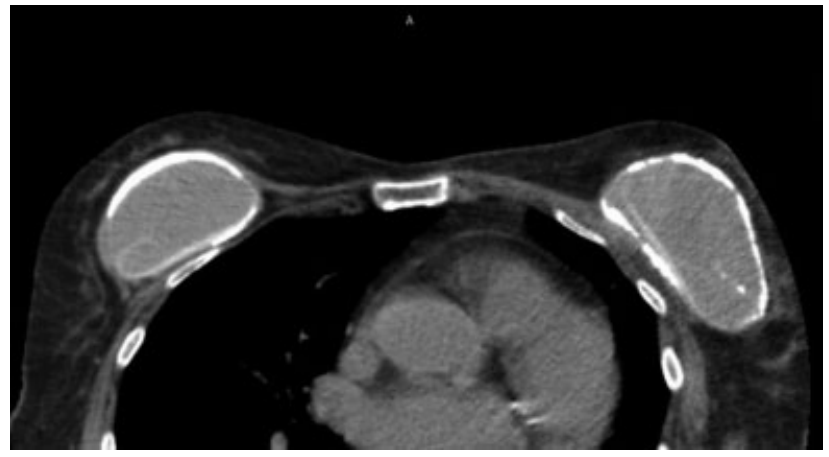

Fig. 6 Severe capsular fibrosis with extensive calcifications in an 83 -year-old women undergoing staging for cancer. Notably there is intracapsular rupture of both implants as well.

formed fibrous capsule around the implant starts to contract, this is leading to deformation of the implant, discomfort, and pain. $^{5}$ Common imaging findings are deformation of the implant, an increased number of infolds and a thickening of the fibrous capsule ${ }^{5}$ ( - Fig. 5). The thickening of the fibrous capsule is usually examined with ultrasound but can be occasionally seen also on CT. In severe cases, calcification of the fibrous capsule occurs (-Fig. 6).

\section{Cancer}

Breast cancer can be found in augmented breasts, the risk of breast cancer is especially increased in patients after breast reconstruction ( - Fig. 7). On CT, breast cancer is shown as a mass with contrast enhancement. Because of the limited resolution, irregularities of the lesion, which are a hallmark of malignancy on mammography, can sometimes be invisible with CT imaging. Therefore solid lesion needs further workup with ultrasound and mammography. Exceptions are lesions with coarse central calcifications, which are pathognomic for involuted fibroadenomas ${ }^{6}$. It has to be noted that microcalcifications cannot be seen with $\mathrm{CT}^{6}$

\section{Systemic Manifestations of Silicone Leakage}

Several pathologies with silicone leakage have been reported. These include silicone thorax, pleural effusions, autoimmune/inflammatory syndromes induced by adjuvants (ASIA), siliconosis, and anaplastic large cell lymphoma. ${ }^{7}$ All pathologies are exceedingly rare. For example, until 2018, only 516 cases of pathologically proven breast implant-associated anaplastic large cell lymphomas have been reported worldwide. ${ }^{8}$ The incidences of these rare complications are unknown and only case reports or small case series are available. ${ }^{7}$ The main imaging finding of breast implant-associated lymphoma is a large amount of fluid around the implant. In these cases, aspiration cytopathology is advised. Another rare entity seems to be silicone implantinduced lymphadenopathy, with a larger series of 18 cases reported. ${ }^{9}$ In these cases, there is marked lymphadenopathy (axillar, as well as hilar, and mediastinal), most often in cases

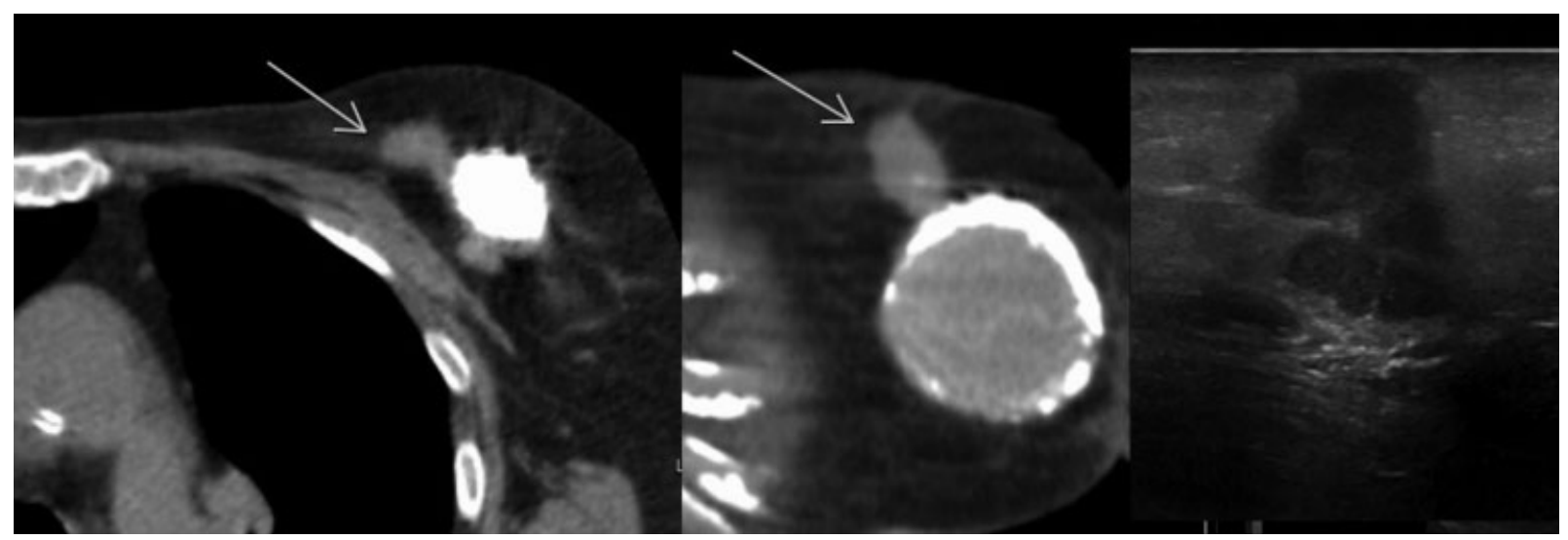

Fig. 7 Breast cancer adjacent to a breast implant in an 83-year-old women undergoing cancer staging. The cancer is shown as a mass with irregular margins and spotted calcifications (arrows). The corresponding sonographic image is shown on the right. 
of an implant rupture. Because there is a wide differential diagnosis including inflammation, malignancies, and granulomatous disease, this is usually a disease exclusion and the diagnosis is often surprisingly made on pathology with the depiction of silicone implants. ${ }^{9}$

\section{Conclusion}

Breast implants are frequently depicted on chest CT performed for various reasons. The knowledge of normal findings and pathology (especially rupture, capsular contracture, and cancer) is crucial to ensure optimal patient outcome.

\section{Conflict of Interest}

There is no conflict of interest to declare.

\section{References}

1 Coombs DM, Grover R, Prassinos A, Gurunluoglu R. Breast augmentation surgery: clinical considerations. Cleve Clin J Med 2019; 86(02):111-122
2 de Boer M, van Middelkoop M, Hauptmann M, et al. Breast implant prevalence in the dutch female population assessed by chest radiographs. Aesthet Surg J 2020;40(02):156-164

3 Juanpere S, Perez E, Huc O, Motos N, Pont J, Pedraza S. Imaging of breast implants-a pictorial review. Insights Imaging 2011;2(06):653-670

4 Medina FM, Calle JA, Garcia Ledesma OE, Echeverry J. Breast implants, adverse events and rare complications: findings by mammography, US and MR imaging. Rev Colomb Radiol. 2016; 27:4434-4440

5 Gossner J. Sonography in capsular contracture after breast augmentation: value of established criteria, new techniques and directions for research. J Ultrasound 2016;20(01):87-89

6 Gossner J. Intramammary findings on CT of the chest- a review of normal anatomy and possible findings. Pol J Radiol 2016; 81:415-421

7 Singh J, Inaty H, Mukhopadhyay S, Mehta AC. Chronic pulmonary silicone embolism from breast augmentation is not a common finding in explanted lungs. Pulm Med 2018;2018:2987072

8 Kricheldorff J, Fallenberg EM, Solbach C, Gerber-Schäfer C, Ransco C, Fritschen UV. Breast impant-associated lymphoma. Dtsch Arztebl Int 2018;115(38):628-635

9 Bauer RP, Krajicek BJ, Daniel CR, Shah SS, Ryu JH. Silicone breast implant- induced lymphadenopathy: 18 cases. Respir Med CME 2011;4:126-130 